$>6$ months' duration and who experienced unsuccessful intercourse on at least $50 \%$ of attempts during a 4-week, treatment-free, runin period. In total, 194 patients were randomly allocated to treatment with vardenafil (at an initial dose of $10 \mathrm{mg}$, adjusted after 2 weeks to 5,10 or $20 \mathrm{mg}$ ) and 189 patients to placebo treatment, to be taken $8 \mathrm{~h}$ before attempting intercourse, for 10 weeks.

Compared with placebo-treated patients, vardenafil-treated patients showed a clinically and statistically significant improvement in the success rate of intercourse attempts $(69 \%$ versus $34 \% ; P<0.001$ ) over weeks $2-10$. Only $4 \%$ of vardenafil-treated men experienced serious adverse events related to the study medication.

The authors call for further studies to determine the full extent of vardenafil's duration of action, and suggest that the pharmacokinetic characteristics of vardenafil are responsible for both its rapid onset and extended duration of action.

Original article Porst $\mathrm{H}$ et al. (2006) Extended duration of efficacy of Vardenafil when taken 8 hours before intercourse: a randomized, double-blind, placebo-controlled study. Eur Urol 50: 1086-1095

\section{Using helical CT to choose the appropriate surgery for RCC}

Minimally invasive and nephron-sparing surgery (NSS) is becoming more and more popular in the treatment of localised renal cell carcinoma, although total and radical nephrectomy still have their place. Accurate knowledge of the tumor position and other tumor characteristics is vital in deciding which type of surgery is right for each patient. For instance, surgeons might decide total nephrectomy is preferable if NSS might lead to injury to the renal collecting system. Derweesh et al. investigated, in a retrospective study, whether information from helical CT can predict collecting-system injury during NSS.

Three-phase renal helical CT scans were obtained for 344 patients (mean age 61 years, 232 males) before they underwent NSS. Collecting-system entry was recorded for 170 of these patients. Collecting-system entry was more common in patients with a central tumor location, calyceal involvement, and tumor size $>4 \mathrm{~cm}$ (all $P \leq 0.005$ ). Nearly three-quarters of the patients with a centrally located tumor experienced collecting-system entry during surgery.

The authors conclude that helical CT is a useful tool for the assessment of tumor characteristics, and might aid the surgeon in choosing the most appropriate type of surgery for individual patients.

Original article Derweesh IH et al. (2006) The predictive value of helical computed tomography for collectingsystem entry during nephron-sparing surgery. BJU Int 98: 963-968

\section{How useful is bladder cancer screening?}

The majority of patients who die from bladder cancer have high-grade, muscle-invasive disease. It would seem logical, therefore, that screening programs that detect bladder cancer before it reaches this stage are useful in lowering mortality rates. A US team has investigated whether bladder cancer screening in Madison, WI, resulted in improved outcomes for screened patients, compared with unscreened patients.

The screening cohort comprised 1,575 men aged at least 50 years recruited from wellpatient clinic registers. These men regularly tested their urine for hematuria at home, using a chemical reagent strip. Men who obtained a positive result underwent a standard urologic examination. Of the 258 screened patients who underwent this examination, 21 were diagnosed with bladder cancer. Tumor characteristics and patient outcomes were compared with those of 509 men in the Wisconsin Tumor Registry who were diagnosed in the same time period.

High-grade superficial or invasive bladder cancer was significantly less common in the screened cohort ( $10 \%$ vs $60 \%, P=0.002)$. At 14 years' follow-up, no screened patients with bladder cancer had died, compared with 104 unscreened patients $(P=0.02)$.

The authors conclude that home reagentstrip testing for hematuria is an effective means of bladder cancer screening, which increases the likelihood of early detection of disease and seems to reduce mortality due to bladder cancer.

Original article Messing EM et al. (2006) Long-term outcome of hematuria home screening for bladder cancer in men. Cancer 107: 2173-2179 